\title{
Ethnologies
}

Lafitau et l'émergence du discours ethnographique. Par Andreas Motsch. (Sillery/Paris : Septentrion et Presses de l’Université de Paris Sorbonne, 2001. Pp. 312, ISBN 2

89448-184-5)

\section{Stéphanie Chaffray}

Volume 26, numéro 1, 2004

Littératie

Literacy

URI : https://id.erudit.org/iderudit/013355ar

DOI : https://doi.org/10.7202/013355ar

Aller au sommaire du numéro

Éditeur(s)

Association Canadienne d'Ethnologie et de Folklore

ISSN

1481-5974 (imprimé)

1708-0401 (numérique)

Découvrir la revue

Citer ce compte rendu

Chaffray, S. (2004). Compte rendu de [Lafitau et l'émergence du discours

ethnographique. Par Andreas Motsch. (Sillery/Paris : Septentrion et Presses de

l'Université de Paris Sorbonne, 2001. Pp. 312, ISBN 2 89448-184-5)]. Ethnologies,

26(1), 256-260. https://doi.org/10.7202/013355ar d'utilisation que vous pouvez consulter en ligne.

https://apropos.erudit.org/fr/usagers/politique-dutilisation/ 
de discourir très longtemps pour saisir l'habileté qu'ont plusieurs d'entre eux à accrocher rapidement leur auditoire :

Once upon a time, an' a very good time it was, not in your time, 'deed in my time, in olden times, when quart bottles hold half a gallon, an' houses were papered with pankakes, an' pigs run about, forks stuck in their ass, see who wanted to buy pork... ( «The Queen of Paradise's Garden » 89)

ainsi que le rythme qu'ils peuvent donner aux contes en utilisant par exemple certaines répétitions et leur maîtrise de l'effet de surprise, qui ne réside souvent pas dans le dénouement de l'histoire, mais bien dans la façon dont ils vont formuler cette conclusion.

Il me semble que cet ouvrage reflète la confiance de l'éditeur en la valeur universelle de ces contes, en leur capacité de vivre d'eux-mêmes, sans trop d'explications. Souhaitons que cette édition puisse, comme le souhaite son éditeur, rejoindre les communautés qui nous ont légué ces contes... et qu'ils continuent de circuler. De nouveaux conteurs s'en feront-ils les porteurs?

Mathieu Perron

Saint-Pascal, Québec

\section{Référence}

Halpert, Herbert et J.D.A Widdowson, 1996, Folktales of Newfoundland. The Resilience of the Oral Tradition. New York et Londres: Garland Publishing.

Lafitau et l'émergence du discours ethnographique. Par Andreas Motsch. (Sillery/Paris : Septentrion et Presses de I'Université de Paris Sorbonne, 2001. Pp. 312, ISBN 2 89448-184-5)

Au cours des premières décennies du XVIII ${ }^{\mathrm{e}}$ siècle, le père jésuite Joseph-François Lafitau séjourna à deux reprises en Amérique du Nord, côtoyant des Iroquois, des Hurons et d'autres nations amérindiennes des environs de Montréal. C'est au terme de son premier voyage (17121717), qu'il entama l'écriture des Mours des Sauvages Américains comparées aux moeurs des Premiers Temps, publié à Paris en 1724. Il y décrit les modes de vie des habitants de l'Amérique en les rapprochant de ceux des peuples de l'Antiquité classique, afin de prouver leur origine commune. 
Lafitau et l'émergence du discours ethnographique, qui est la thèse de doctorat en littérature comparée qu'Andreas Motsch a soutenue à l'Université de Montréal en 1995, propose une relecture de ce célèbre texte qui valut à son auteur d'être considéré comme le père de l'ethnographie comparative. Les recherches d'Andreas Motsch portent sur la théorie et l'épistémologie de la littérature, l'émergence de la modernité dans ses rapports avec l'Autre, l'analyse du discours ainsi que les théories de l'altérité et de la production de savoir. Il fait montre d'un intérêt marqué pour la littérature de contact, sur laquelle il a publié plusieurs articles. Il est actuellement professeur de littérature à l'Université de Toronto.

Lafitau et l'émergence du discours ethnographique ne porte pas sur le contenu ethnographique de l'ouvrage de Lafitau - contenu d'un intérêt au demeurant indéniable. Ce qui intéresse Andreas Motsch, c'est plutôt de mettre en évidence l'émergence discursive de ce savoir, de comprendre ses fondements épistémologiques et ses conditions de possibilité, afin de produire, comme dans la tradition foucaldienne, une archéologie du savoir. Cette démarche permet de montrer que le texte de Lafitau constitue une ouvre ethnographique avant la lettre, que c'est un texte d'une étonnante modernité, contenant les prémices de problèmes débattus par l'anthropologie actuelle. Si les spécialistes qui se sont penchés sur les conséquences économiques et politiques de la découverte de l'Amérique sont nombreux, ceux qui se sont interrogés sur les conséquences et les questionnements épistémologiques liés à cette nouveauté le sont moins. Ce sont ces aspects - moins connus qu'Andreas Motsch aborde dans cet ouvrage. La découverte de l'Amérique a provoqué un vaste projet d'écriture, obligeant les Européens à mettre par écrit ce qu'ils percevaient comme différent. Dans la littérature de contact, l'écriture est donc à la fois le témoin de la confrontation avec l'autre et le lieu même de cette confrontation. Elle constitue un espace où les Européens affirment leur identité et fabriquent celle de l'autre. Andreas Motsch entend ainsi rétablir le lien entre l'auteur et son texte et mettre en lumière la logique qui sous-tend le projet des Mours des Sauvages Américains.

L'organisation du texte répond à une volonté de déconstruire le discours de Lafitau afin de dégager les dynamiques qui l'animent. Après avoir dégagé les buts épistémologiques et politiques de Lafitau, Andreas Motsch analyse la solution proposée par le père jésuite en décrivant les règles et les figures discursives de cette solution. 
Le premier chapitre traite du contexte de parution de l'ouvrage et de ses influences sur l'écriture. Le début du siècle des Lumières était encore marqué par les bouleversements épistémologiques et politiques provoqués par l'Humanisme d'une part et par la Réforme d'autre part, qui avaient entraîné une crise de l'Église catholique en remettant en cause et son autorité et son fondement. La Compagnie de Jésus s'était engagée au cours du XVII ${ }^{\mathrm{e}}$ siècle à défendre ardemment la ContreRéforme, en s'efforçant de rétablir l'autorité interprétative de l'Église et de refaire sous sa coupe l'unité du monde chrétien. Le projet de Lafitau s'inscrit au XVIII ${ }^{\mathrm{e}}$ siècle dans le prolongement de cette action. Il vise un public sceptique, voire athée, qu'il tente de convaincre en élaborant une vision du monde qui réconcilierait les principes de la religion avec les nouvelles vérités rationalistes. Le projet de Lafitau comporte deux volets interdépendants, l'un épistémologique, l'autre politique. Le cadre épistémologique s'articule autour de l'idée que tous les hommes, y compris les habitants de l'Amérique, ont pour origine Adam et Eve. Le projet politique repose sur l'argument que la religion est la source de toute pratique humaine, ce qui justifie l'autorité de l'Église catholique auprès des gouvernements européens. Ces deux postulats trouvent leur fondement dans la similitude entre les Américains et les anciens peuples de l'Antiquité occidentale. Cette comparaison n'est finalement qu'un prétexte pour rapatrier les Amérindiens dans la création divine, réaffirmer l'universalité de la religion et rétablir l'autorité de l'Église catholique pour interpréter le monde. Même si Lafitau revalorise l'image souvent négative des Amérindiens et offre une alternative à l'habituelle comparaison avec les Tupinamba du Brésil, il les enferme dans une autre altérité, marquée par ses présupposés religieux. Le discours sur l'Autre se résume en réalité à un discours du même. L'Amérique n'est alors qu'un laboratoire servant à réaliser les utopies politiques de l'Église.

Le second chapitre décrit le cadre ontologique de la description ethnographique. Andreas Motsch y analyse de quelle manière Lafitau aborde les thèmes du temps, de l'espace et de l'agencement. Au lieu de reconnaître la spécificité de la perception du temps et de l'espace par les Amérindiens, il les ramène à des conceptions occidentales. Le temps est perçu comme une unité objective, l'espace comme un espace physique inanimé. Il nie la relation spirituelle que les Amérindiens entretiennent avec l'espace en le réduisant à un rapport de subsistance et de dépendance matérielle. Grâce à cette démonstration, il aboutit à 
une vision globale du monde qui permet de rétablir l'unité de la création divine. L'altérité amérindienne n'est intelligible que dans le cadre des Premiers Temps, ce qui aboutit à nier la contemporanéité des Amérindiens et fait de l'ethnographe l'herméneute par excellence, le témoin de la différence.

Après avoir énoncé le cadre de l'œuvre, Andreas Motsch étudie dans un troisième et dernier chapitre la manière dont le père Lafitau décrit le fonctionnement de la société amérindienne. Il avance l'hypothèse que le fonctionnalisme et l'utilitarisme structurent la pensée et le texte de l'ethnographe et met en évidence la dualité entre la conception utilitariste de l'Occident et l'économie du don qui régit les cultures amérindiennes. Dans la pensée de Lafitau, l'économie du don est interprétée dans un sens chrétien, c'est-à-dire comme un processus fonctionnaliste. Le don est évalué selon sa valeur utilitaire. Un aspect particulièrement pertinent de l'argumentation d'Andreas Motsch est l'étude des différentes formes de don à laquelle il intègre la question de l'échange des femmes, de la monnaie et de l'écriture, en montrant comment dans chacune d'entre elles s'exerce le fonctionnalisme religieux. Le thème de l'économie du don prouve la modernité du texte de Lafitau, puisque ses principes y sont énoncés avant la théorie de Marcel Mauss. Lafitau est aussi le premier à considérer le wampum et le tatouage non pas comme des rites barbares, mais comme des formes d'écriture et de mise en récit de l'histoire.

Andreas Motsch conclut sa démonstration en remarquant qu'en raison de ses présupposés théologiques, le discours de Lafitau n'est pas entièrement rationnel. Cependant, il a indéniablement fourni des informations nouvelles sur cette altérité, ce qui fait de lui le fondateur de l'ethnographie moderne. Il remarque aussi très justement que l'intérêt du texte réside avant tout dans la résistance de l'autre au discours ethnographique.

Andreas Motsch, par une approche multidisciplinaire, parvient à faire une analyse épistémologique convaincante du discours de Lafitau. Il met ainsi en lumière des aspects de cette œuvre jusque là peu abordés par les historiens, les littéraires et les anthropologues. En liant l'approche historique à l'approche littéraire, il réussit à faire ressortir les motivations sous-jacentes de l'auteur et à faire comprendre comment s'opère la mise en discours. 
Cette approche mériterait d'être appliquée à d'autres textes de la littérature de contact, ce qui permettrait d'éclairer le rapport très intéressant entre le concept de l'altérité et la politique coloniale.

Stéphanie Chaffray CÉLAT et Université Paris IV Sorbonne

Collections - Collectionneurs. Textiles d'Amérique et de France. Par Jocelyne Mathieu et Christine Turgeon, dirs. (Québec : Célat et Presses de I'Université Laval, 2002. Pp. 240, ISBN 2-7637-7856-9)

Le fil de l'art. Les broderies des Ursulines de Québec. Par Christine Turgeon (Québec : Musée du Québec et Musée des Ursulines, 2002. Pp. 155, ISBN 2-551-21530-7)

« ... filé plein, lame, filé riant, point lancé empiétant polychrome »; " lampas fond satin blanc, broché soie, chenille, filé et frisé or »; ou encore «velours ciselé deux-corps », «broderies au passé plat, point de tige et point de sable polychrome ${ } . .$.

Si ce n'était que pour le vocabulaire délicat des « artistes à l'aiguille », la finesse d'exécution, voire les prouesses techniques qui se découvrent au fil des broderies anciennes, l'ouvrage CollectionsCollectionneurs vaudrait déjà bien le détour. Mais les désignations si fines de cet ouvrage spécifiquement voué aux textiles, des parements d'autels aux teintures, des courtepointes aux dentelles, nous font aussi entrer de plain-pied dans une petite partie du vaste monde que l'on appelle familièrement les «arts déco ».

Dans les musées, les collections de textiles, volumes gigantesques de pièces fragiles, entre le beau et l'utile, au carrefour des objets d'art et des objets ethnographiques (si tant est qu'il faille distinguer entre les deux, ce que cet ouvrage se garde de faire), soulèvent des questions particulières, en Amérique du Nord et en France. Le problème le plus crucial en ce domaine est que les collections, particulièrement volumineuses, se trouvent être très insuffisamment inventoriées et documentées.

Cet ouvrage, dirigé par Jocelyne Mathieu et Christine Turgeon, confronte les questionnements qui découlent de ce problème essentiel sur une grande échelle. Ce sont les actes du colloque éponyme, 